\title{
The Creation of Desired Microstructures by Internal Oxidation
}

\author{
I. Anzel, ${ }^{*}$ L. Kosec, ${ }^{* *}$ A. C. Kneissl, ${ }^{* * *}$ \\ *Faculty of Mechanical Engineering, University of Maribor, Smetanova 13, 2000 Maribor, Slovenia \\ **Department of Materials and Metallurgy, Faculty of Natural Sciences and Engineering, University \\ of Ljubljana, Aškerčeva 12, SI-1000 Ljubljana, Slovenia \\ ***Department of Physical Metallurgy and Materials Testing, University of Leoben, Franz Josef \\ Strasse 18, A 8700 Leoben, Austria
}

The mechanical behaviour of materials is mainly dependent on the microstructure. By inducing suitable microstructural changes it is possible to optimise the quality or to attain the appropriate combination of properties of constructional and functional materials. There are numerous processes such as plastic deformation, heat treatment, or surface engineering suitable for the creation of desired changes in the material's microstructure. For this purpose also the internal oxidation process as a special case of high temperature oxidation of metallic materials can be used.

Internal oxidation is usually a diffusion-controlled process involving selective reactions of a less noble solute or second phase particles with oxygen (also nitrogen or carbon) diffusing in from the surface (Fig. 1). The phenomenon is well-understood for single phase solid solutions and can be interpreted according to the known theoretical models [1]. From a technological standpoint the process can be used for dispersion hardening of metals and alloys or for obtaining the appropriate combination of electrical and mechanical properties. Contrary to this, the internal oxidation of twophase alloys is a very complex process governed by different mechanisms [2]. These differences originate from the fact that the reactive element, which has a higher affinity to oxygen than the base metal, is distributed between the solid solution-matrix and the second phase (for example: the intermetallic particles). Due to this fact, many competing processes - simultaneously or subsequently, take place during the internal oxidation of such an alloy. Because the alloys of two or more phases represent a class of materials present in many commercial applications it is of great importance to understand their high temperature oxidation.

Over the last few years also the activities in the field of studying the internal oxidation in thermodynamically metastable metallic materials (for example: rapidly solidified parts or thin surface layers) have increased. Their microstructures are mostly unstable at higher temperatures and they transform very rapidly into more stable states. However, specific chemical reactions can also take place in such systems. For instance, the oxygen atoms that dissolve in the rapidly solidified pure metals during the high temperature annealing, concentrate at the defects in the metal crystal lattice and produce high local supersaturations. The increasing oxygen activities at these places cause the local oxidation and consequently the precipitation of fine oxide particles - paradox: internal oxidation of pure metal (Fig. 2). In the proposed paper the cases of some specific internal oxidation processes and the influence on the microstructure and properties will be presented.

\section{References}

[1] C. Wagner, Z. Electrochem. 63 (1959) 772.

[2] F. Gesmundo and B. Gleeon, Oxidation of Metals 44 (1995) 211. 

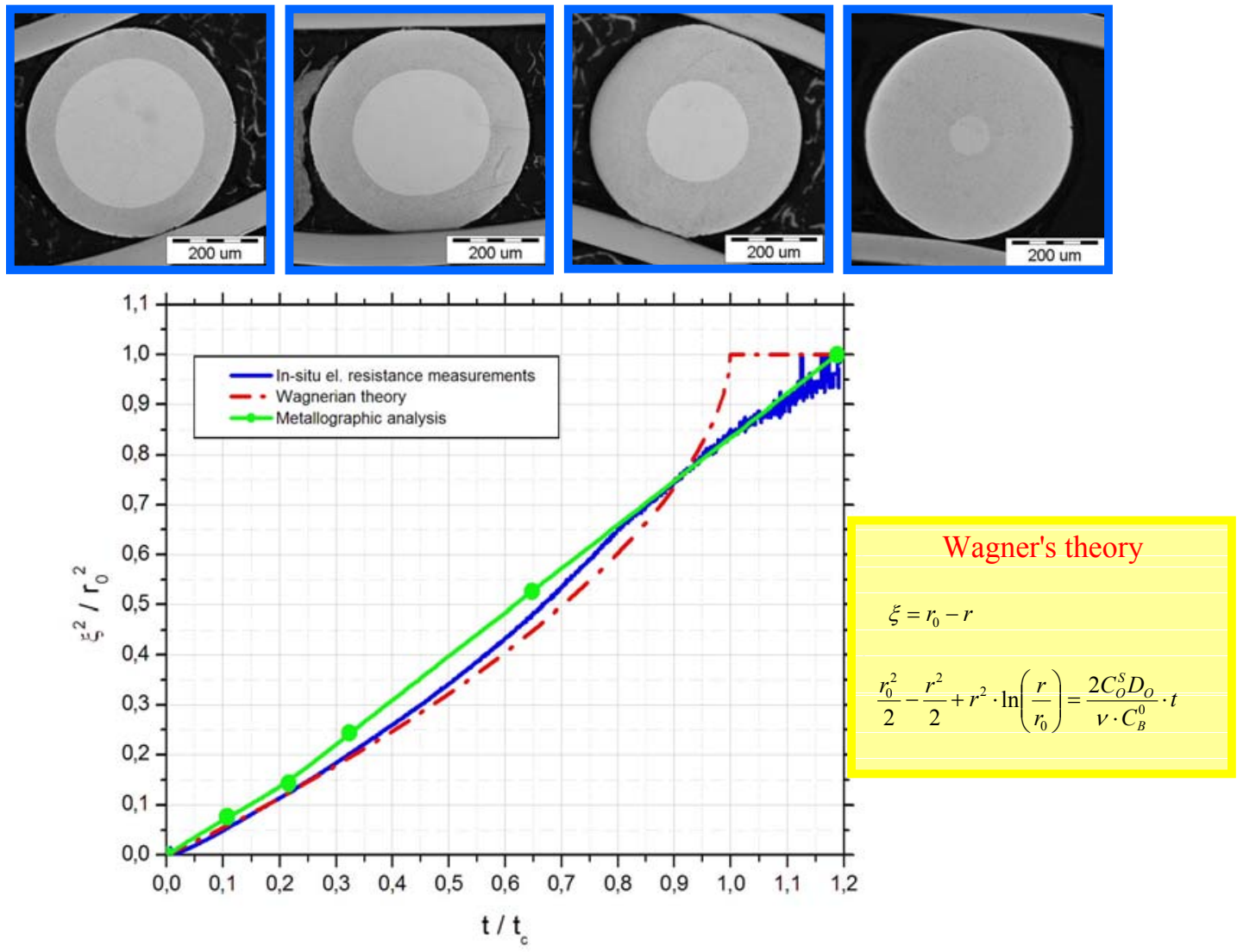

Fig. 1: Comparison of kinetics of internal oxidation of $\mathrm{Ag}-\mathrm{Sn}(2$ at. \% $\mathrm{Sn}$ ) alloy determined by different methods

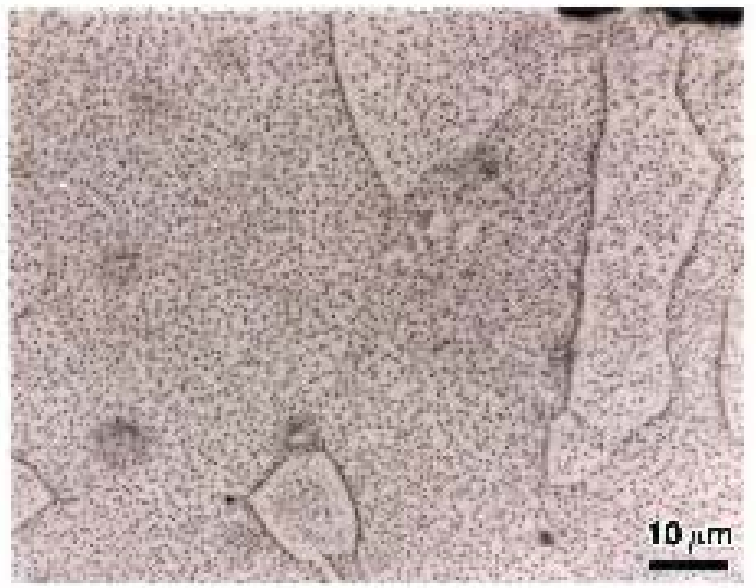

Fig. 2: Optical micrograph (in vertical cross-section) of internally oxidized rapidly solidified ribbon of pure copper after $2 \mathrm{~h}$ at $1173 \mathrm{~K}$. 